\section{Validade de indicadores de atividade física e comportamento sedentário da Pesquisa Nacional de Saúde do Escolar entre adolescentes do Rio de Janeiro, Brasil}

\author{
Validity of indicators on physical activity and \\ sedentary behavior from the Brazilian National \\ School-Based Health Survey among adolescents in \\ Rio de Janeiro, Brazil
}

\author{
Validez de indicadores de actividad física y \\ conducta sedentaria de la Encuesta Nacional de \\ Salud Escolar entre adolescentes en \\ Río de Janeiro, Brasil
}

\begin{abstract}
This study evaluated the relative validity of physical activity indicators from the questionnaire used in the Brazilian National SchoolBased Health Survey (PeNSE) in the city of Rio de Janeiro, Brazil, based on a sample of $174 \mathrm{stu}$ dents. The following indicators of weekly physical activity were evaluated: ACTIVE-300MIN ( $\geq 300$ minutes/week); ACTIVE-150MIN ( $\geq 150$ minutes), INACTIVE (no physical activity). Additionally, indicators of sedentary behavior were also assessed, as daily screen time (TV, videogames, and computer). The results from the questionnaire were compared with three 24-hour recalls. The results of ACTIVE-300MIN, ACTIVE-150MIN, and INACTIVE generated by PeNSE showed high accuracy. These indicators performed better than those of sedentary behavior in relation to frequency estimates as well as sensitivity, specificity, and correct classification rate. The indicators of physical activity from PeNSE showed satisfactory relative validity.
\end{abstract}

Motor Activity; Validation Studies; Sedentary Lifestyle; Adolescent; Surveillance
Letícia Ferreira Tavares 1

Inês Rugani Ribeiro de Castro 1

Letícia Oliveira Cardoso 2

Renata Bertazzi Levy 3

Rafael Moreira Claro 4

Andreia Ferreira de Oliveira 5

\title{
Resumo
}

Analisou-se a validade relativa dos indicadores de atividade física do questionário utilizado na Pesquisa Nacional de Saúde do Escolar (PeNSE) entre adolescentes da cidade do Rio de Janeiro, Brasil. Foram estudados 174 alunos. Avaliaram-se os seguintes indicadores referentes ao tempo semanal acumulado com a prática de atividade física: ATIVO-300MIN (acumulou 300 minutos ou mais); ATIVO-150MIN (acumulou 150 minutos ou mais); INATIVO (não praticou atividade física). Também foram estudados os indicadores de comportamento sedentário referentes ao tempo diário despendido assistindo TV, jogando videogame e usando computador. Foram comparados os resultados do questionário e dos três recordatórios de 24 horas. Os resultados de ATIVO-300MIN, ATIVO-150MIN e INATIVO gerados pela PeNSE apresentaram alta acurácia. Esses indicadores tiveram melhor desempenho que os de comportamento sedentário, tanto em relação às estimativas de frequência quanto em relação à sensibilidade, especificidade e índice de acurácia. Os indicadores de atividade física da PeNSE apresentaram validade relativa satisfatória.

Atividade Motora; Estudos de Validação; Estilo de Vida Sedentário; Adolescente; Vigilância 


\section{Introdução}

Em qualquer idade, a prática de atividade física é uma atitude essencial para melhorar a saúde física e mental dos indivíduos 1. Entre crianças e adolescentes, níveis apropriados de atividade física aumentam a consciência neuromuscular (coordenação e controle dos movimentos), o desenvolvimento do sistema cardiovascular e a saúde musculoesquelética, além de contribuírem para a manutenção do peso corpóreo saudável ${ }^{2}$. Além dos benefícios em curto prazo, a prática de atividade física na adolescência influencia os níveis de atividade física nos anos seguintes e está diretamente relacionada com o surgimento de morbidades e com a mortalidade na vida adulta 3 .

Ainda que seja reconhecida a importância da prática de atividade física na adolescência, em diversas partes do mundo, os níveis de inatividade física são elevados nesse grupo populacional, com implicações no estado geral de saúde e no aumento do risco de ocorrência de doenças crônicas não transmissíveis 4,5,6,7,8. Some-se a isso a alta prevalência, nesse grupo, de comportamentos sedentários - como assistir televisão e usar videogame e computador - associada à obesidade, ao tabagismo e ao colesterol elevado 6,7,8,9,10,11.

Diversos países têm implementado sistemas de vigilância de fatores de risco e proteção associados às doenças crônicas não transmissíveis específicos para adolescentes que incluem, entre outros temas, questões referentes às práticas de atividade física e de lazer sedentário 12,13,14. No Brasil, a Secretaria de Vigilância em Saúde do Ministério da Saúde, em parceria com o Ministério da Educação e com o Instituto Brasileiro de Geografia e Estatística (IBGE), estruturou um sistema de vigilância de fatores de risco para a saúde de adolescentes baseado em inquéritos regulares realizados em escolas, denominados Pesquisa Nacional de Saúde Escolar (PeNSE). A primeira edição da PeNSE foi realizada no ano de 2009 em uma amostra probabilística de adolescentes do 9o ano do Ensino Fundamental de escolas públicas e privadas das 26 capitais de estados brasileiros e do Distrito Federal 15. A coleta de dados da segunda edição da PeNSE ocorreu no primeiro semestre de 2012.

A atividade física e o comportamento sedentário são eventos de difícil aferição. Métodos diretos, como o pedômetro e o acelerômetro, e métodos indiretos, como questionários, recordatórios e registros, têm sido utilizados para este fim ${ }^{3,10}$. Uma questão a ser considerada é a carência de instrumentos considerados válidos e operacionalmente viáveis de serem aplicados em inquéritos populacionais como os realizados pelos sistemas de vigilância.

A aferição acurada de eventos analisados por meio de indicadores empregados por um sistema de vigilância depende da validade e da confiabilidade do instrumento utilizado na coleta de dados 16. Em âmbito internacional, há poucos estudos que avaliam a validade dos indicadores da prática de atividade física utilizados pelos sistemas de vigilância específicos para adolescentes 17,18,19,20, não tendo sido encontrado nenhum trabalho sobre os indicadores relacionados aos comportamentos sedentários. Até o momento não foram realizados estudos de validação do questionário utilizado pelo sistema de vigilância de fatores de risco para a saúde de adolescentes brasileiros. O presente trabalho tem como objetivo analisar a validade relativa dos indicadores do módulo de atividade física e lazer sedentário do questionário utilizado na PeNSE.

\section{Métodos \\ População do estudo e amostragem}

A população de estudo foi composta por alunos que cursavam, em 2011, o 9o ano do Ensino Fundamental em escolas públicas e privadas da cidade do Rio de Janeiro.

Os resultados obtidos em estudo de reprodutibilidade do questionário utilizado pelo sistema de monitoramento dessa cidade, realizado em 2007 com alunos do último ano do Ensino Fundamental de escolas públicas, serviram de base para a definição do tamanho amostral do presente trabalho 11. A amostra foi dimensionada considerando-se o menor valor de kappa observado $(0,32$ para a questão referente à frequência de consumo de alimentos embutidos), a prevalência de consumo frequente (pelo menos cinco dias na semana) deste grupo de alimentos (20\%), precisão absoluta de 0,20 e nível de 95\% de confiança, totalizando 126 alunos. O cálculo foi realizado implementando-se a rotina sample size for the kappa-statistic measure of interrater agreement (sskdlg) no programa Stata, versão 10 (Stata Corp., College Station, Estados Unidos) 16,21. Adotou-se uma amostra inicial de 250 alunos, assumindo-se um porcentual de perdas de $50 \%$ na segunda etapa do estudo (três entrevistas por meio de contato telefônico).

Dados do Censo Escolar 2010, disponibilizados pelo Instituto Nacional de Estudos e Pesquisas Educacionais Anísio Teixeira/Ministério da Educação (INEP/MEC) foram utilizados como base para sorteio e seleção das turmas. Das 3.352 escolas existentes no município, 1.093 
tinham turmas de 9o ano. Foram excluídas 152 escolas consideradas não elegíveis por apresentarem turmas de 9o ano com número inferior a 15 alunos. De acordo com o Censo Escolar 2010, as turmas de escolas públicas e privadas tinham em média 36,2 e 25,7 alunos, respectivamente, sendo necessário, portanto, a seleção de aproximadamente nove turmas para atingir o tamanho amostral. A distribuição do número de turmas por dependência administrativa da escola tomou por base a proporção de alunos matriculados em escolas públicas (75\%) e privadas (25\%) do Município do Rio de Janeiro.

O desenho amostral compreendeu um único estágio: turmas. A seleção das turmas se deu por meio de amostragem aleatória e sistemática, e o intervalo de sorteio foi calculado de acordo com o número total de turmas do 9o ano das escolas públicas e privadas. Adicionalmente, foram sorteadas três turmas de diferentes escolas para reposição no mesmo bairro, para o caso de não ser possível realizar o estudo com a turma inicialmente selecionada. Quando não havia número suficiente de escolas para a reposição no bairro, esta reposição foi prevista com escolas de bairro vizinho. Todos os alunos das turmas sorteadas foram convidados a participar do estudo. Foram sorteadas nove turmas distribuídas em nove escolas (seis públicas e três privadas) espalhadas aleatoriamente pela cidade do Rio de Janeiro.

As escolas onde se encontravam as nove turmas sorteadas aceitaram participar do trabalho. O principal motivo de não realizar a segunda etapa completa do estudo (três recordatórios de 24 horas) foi não conseguir contatar os alunos por telefone. Foi avaliado um total de 174 alunos (Figura 1).

\section{Coleta de dados}

A primeira etapa do estudo consistiu na realização de visita às escolas para preenchimento do questionário (método teste, detalhado a seguir). Com o intuito de reproduzir as operações realizadas durante a coleta de dados da PeNSE, os adolescentes responderam ao questionário completo, ainda que o presente estudo tenha sido direcionado exclusivamente ao módulo referente à atividade física. O preenchimento do questionário foi feito utilizando-se microcomputador de mão (personal digital assistant - PDA) da mesma versão adotada na edição de 2012 da PeNSE.

Posteriormente, foram realizadas as ligações telefônicas para a realização dos recordatórios de 24 horas - R24h (método de referência, detalhado a seguir) - em dias alternados, sendo dois recordatórios referentes a dias de semana e um referente a um dia de final de semana. Foi criado um cronograma de ligações que garantiu que todos os dias da semana fossem contemplados proporcionalmente no estudo. Caso não fosse possível o contato com o adolescente na data prevista para a aplicação de algum dos três recordatórios, novo contato era realizado no mesmo dia da semana seguinte. A finalização dessa etapa (três R24h) ocorreu no máximo 15 dias após a aplicação do QUEST. A coleta de dados foi realizada de setembro a novembro de 2011 e durou 51 dias.

\section{QUEST (método teste)}

Os assuntos contemplados no questionário utilizado pela PeNSE e, portanto, contidos no QUEST foram: características sociodemográficas, alimentação, imagem corporal, atividade física, tabagismo, consumo de álcool e outras drogas, saúde bucal, comportamento sexual, violência, acidentes, segurança, dados antropométricos (autorreferidos) e apreciação geral do questionário. O questionário da PeNSE pode ser acessado na íntegra na página de Internet do IBGE (http://www.ibge.gov.br/home/estatistica/po pulacao/pense/questionario.pdf, acessado em 12/Dez/2013).

Avaliou-se a atividade física praticada nos sete dias anteriores à pesquisa, levando-se em conta o deslocamento para a escola (ida e volta), a aula de educação física realizada na escola, a prática de atividade física durante o recreio ou intervalo, a realização de atividade física com orientação profissional (sem contar a aula de educação física) e a realização de atividade física sem orientação profissional. A exposição a comportamentos sedentários foi avaliada com base no número de horas em um dia de semana comum (segunda a sexta-feira) despendidas ao assistir televisão, jogar videogame e usar computador.

Nesse instrumento, um exemplo de estrutura de pergunta foi: “Nos últimos 7 dias, quanto tempo por dia você fez atividade física ou esporte durante as aulas de educação física na escola?" e as opções de resposta foram: "não fiz aula de educação física na escola”, "menos de 10 minutos por dia”, "10 a 19 minutos por dia”, "20 a 29 minutos por dia”, “30 a 39 minutos por dia”, “40 a 49 minutos por dia”, “50 a 59 minutos por dia” e “60 minutos ou mais por dia”.

\section{R24h (método de referência)}

Foram aplicados três R24h sobre atividade física e comportamentos sedentários por meio de entrevista telefônica realizada em dias alternados, sendo dois recordatórios referentes a dias de semana e um referente a um dia de final de semana. Para o preenchimento do R24h, o pes- 
Fluxograma de participação no estudo de validação dos indicadores de atividade física da Pesquisa Nacional de Saúde do Escolar (PeNSE). Escolares do 9o ano no Município do Rio de Janeiro, Brasil, 2011.

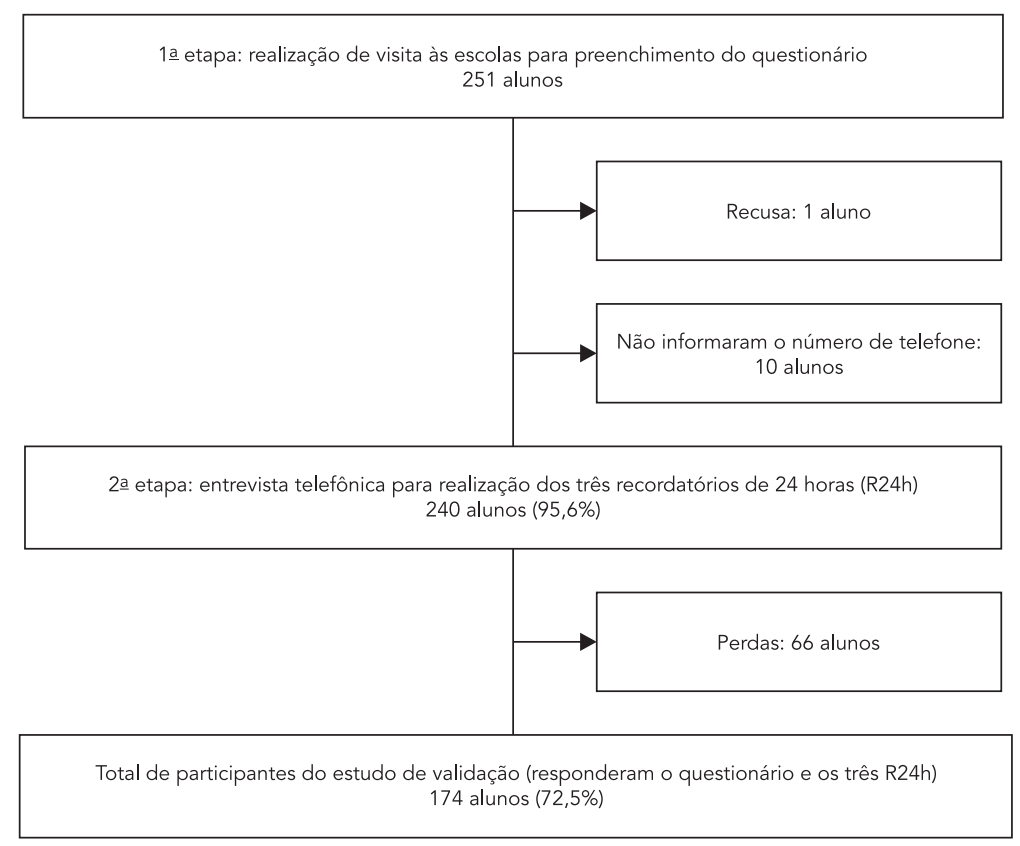

quisador perguntou explicitamente sobre as atividades de interesse (incluídas no questionário da PeNSE) no dia anterior à ligação, desde a hora em que acordou até a hora em que foi dormir 22.

A prática de atividade física na semana anterior à do estudo foi avaliada com base na frequência semanal e duração de cada atividade avaliada, estimando-se o total de minutos acumulados nestas atividades. Foram levados em conta todos os domínios de atividade física avaliados na PeNSE. Para extrapolar os dados obtidos por meio dos três R24h para o total de minutos em uma semana, o tempo despendido em cada atividade física obtido nos dois recordatórios referentes aos dias de semana foi multiplicado por 2,5 (de forma que juntos, representarem os cinco dias) e o recordatório referente ao dia de fim de semana multiplicado por 2,0 (de forma a representar sábado e domingo). O somatório desses valores foi considerado como o tempo semanal total despendido na realização de atividade física.

Para avaliar a exposição a comportamentos sedentários (tempo assistindo TV, jogando videogame e usando computador) foram considerados apenas os R24h referentes aos dias de segunda a sexta-feira, pois a pergunta do questionário da
PeNSE refere-se a um dia da semana comum (ou seja, não leva em consideração o fim de semana). Como método de referência, foram adotadas alternativamente duas formas de estimar o tempo despendido em comportamentos sedentários: (a) a condição ter sido atendida em pelo menos um dos dois R24h (por exemplo: ter assistido TV por duas horas ou mais em pelo menos um dos dois R24h) e (b) a condição ter sido atendida nos dois R24h (por exemplo: ter assistido TV por duas horas ou mais nos dois R24h).

\section{Análise dos dados}

Foram avaliados os seguintes indicadores referentes à prática de atividade física na semana anterior ao estudo: ATIVO-300MIN (acumulou 300 minutos ou mais de atividade física); ATIVO150MIN (acumulou 150 minutos ou mais de atividade física); INATIVO (não praticou qualquer tipo de atividade física) 23 . Os pontos de corte de 300 e 150 minutos por semana foram adotados por expressarem a recomendação internacional de atividade física para adolescentes e adultos, respectivamente 1 . 
Os indicadores de comportamento sedentário adotados tiveram como base o número de horas diárias alocadas nestas atividades, com ponto de corte de duas ou mais horas/dia: tempo assistindo TV-2h, tempo jogando videogame$2 \mathrm{~h}$ e tempo usando computador-2h 4,23 . Para diferenciar as duas formas de construção desses indicadores, no método de referência, ao final do nome de cada um dos indicadores de comportamento sedentário, foi acrescentada a letra "a”, quando a condição foi atendida em pelo menos um dos R24h, e a letra "b", quando a condição foi atendida nos dois R24h, por exemplo: tempo assistindo TV-2ha e tempo assistindo TV-2hb.

O estudo de validade consistiu em comparar os indicadores de atividade física e de comportamentos sedentários obtidos usando-se o QUEST (método teste) e os R24h (método de referência). Com o objetivo de identificar se houve seletividade entre os adolescentes não avaliados foram comparadas as variáveis: idade, índice de massa corporal (IMC), escolaridade materna, sexo e dependência administrativa da escola das populações avaliada e não avaliada aplicando-se os testes Mann-Whitney (para idade), teste t (para IMC) e qui-quadrado (para as outras três variáveis). Todas as análises foram realizadas para o total da amostra e, separadamente, por sexo e por dependência administrativa da escola (pública ou privada). Utilizou-se o teste McNemar para comparar a diferença entre as proporções estimadas pelos dois instrumentos, com nível de significância de 0,05.

Assumindo-se que a verdadeira condição dos indivíduos foi conhecida por meio dos R24h, para avaliar a capacidade do QUEST de classificá-los corretamente segundo sua prática de atividade física e comportamento sedentário foram estimadas a sensibilidade (capacidade de classificar como positivo quem de fato apresenta a condição de interesse) e a especificidade (capacidade de classificar como negativo quem de fato não apresenta a condição de interesse) de cada indicador. Para sumarizar a concordância obtida para cada indicador calculou-se o índice de acurácia do método teste, que é estimado por meio da proporção de classificações corretas (verdadeiros positivos e verdadeiros negativos) entre os indivíduos estudados. Esse índice permite observar o desempenho dos indicadores de maneira global e parece ser um bom parâmetro para avaliar o desempenho de indicadores no contexto de sistemas de vigilância, uma vez que estes pretendem avaliar os fatores de risco e proteção à saúde no âmbito coletivo 24 .

Adicionalmente, foi comparada a mediana de minutos despendidos por semana com a prática de atividade física, obtida com base nos três R24h dos indivíduos classificados pelo QUEST como positivo ("caso") e negativo ("não caso") para os indicadores ATIVO-300MIN, ATIVO-150MIN e INATIVO. Dado que se constatou que a variável tempo semanal total despendido na realização de atividade física não apresentava distribuição normal na amostra estudada, optou-se por utilizar o teste não paramétrico de Wilcoxon para a comparação de medianas dos grupos (positivo e negativo) 25 . Em caráter complementar, também foram realizados os procedimentos propostos por Bland \& Altman 26,27 para avaliar a concordância das estimativas do tempo total despendido com a prática de atividade física segundo o QUEST e os três R24h.

As análises estatísticas foram realizadas com o auxílio do SPSS, versão 17 (SPSS Inc., Chicago, Estados Unidos) e do Microsoft Office Excel 2007 (Microsoft Corp., Estados Unidos).

\section{Aspectos éticos}

O trabalho foi aprovado pelo Comitê de Ética em Pesquisa da Secretaria Municipal de Saúde e Defesa Civil do Rio de Janeiro, sob protocolo de pesquisa no $138 / 10$. A participação dos alunos foi voluntária, facultando-se a possibilidade de desistência em qualquer etapa do estudo. Assim como na PeNSE, o termo de consentimento livre e esclarecido de participação foi preenchido pelo próprio aluno na tela do PDA.

\section{Resultados}

Do total de 174 alunos avaliados, 54\% eram do sexo feminino e $67,2 \%$ estudavam em escola pública. A faixa etária do grupo estudado variou de 13 a 17 anos, com média de idade de 14,7 anos (14,9 anos entre alunos de escolas públicas e 14,5 anos entre aqueles de escolas particulares). Houve diferença estatisticamente significativa ( $\mathrm{p}=$ 0,017 ) entre o grupo estudado e o não estudado em relação à dependência administrativa da escola, com maior proporção de adolescentes de escola privada entre os não estudados $(36,7 \%$ contra $22 \%$ nas escolas públicas).

Em relação ao tempo semanal acumulado com a prática de atividade física, houve diferença estatisticamente significativa entre as estimativas dos dois métodos de aferição apenas para o indicador INATIVO, com valor inferior para a estimativa obtida pelo método teste. Exceto para o indicador tempo assistindo TV-2ha, houve diferença estatisticamente significativa entre as estimativas obtidas pelo QUEST e pelos R24h para todos os indicadores de comportamento seden- 
tário, com proporções sempre mais altas para as estimativas obtidas pelo primeiro. Para ambos os grupos de indicadores (atividade física e comportamento sedentário) foram observados, em geral, valores mais altos para sensibilidade do que para especificidade. Para o conjunto de indicadores, a sensibilidade variou de $8,3 \%$ a $100 \%$, a especificidade de $29 \%$ a $98,7 \%$ e o índice de acurácia de $55,7 \%$ a $92,4 \%$ (Tabela 1 ). Resultado similar foi observado quando utilizados outros pontos de corte ( $\geq 4 \mathrm{~h} /$ dia e $\geq 6 \mathrm{~h} /$ dia) para os indicadores de comportamento sedentário (dados não apresentados).

Em linhas gerais, ao analisar os resultados segundo sexo, assim como observado para o conjunto do grupo estudado, foi observado para ambos os sexos maior acurácia do QUEST na mensuração dos indicadores relacionados à prática de atividade física quando comparados aos de comportamento sedentário. Foram mantidos os resultados dos indicadores ATIVO-300MIN e ATIVO-150MIN observados para o conjunto do grupo estudado. Já para o indicador INATIVO, em contraste com o observado para o conjunto total estudado, entre as meninas não foi observada diferença estatisticamente significativa entre as proporções estimadas pelos dois métodos. Não foi possível realizar a mesma análise estatística para o sexo masculino, pois não houve casos de meninos classificados como inativos segundo o QUEST (Tabela 2).

Em relação aos indicadores de comportamento sedentário, os resultados foram mantidos na análise segundo sexo, com exceção do indicador tempo usando computador-2ha, para o qual não houve diferença estatisticamente significativa entre as estimativas de proporções estimadas pelos dois instrumentos para ambos os sexos. Também os resultados de maior sensibilidade foram mantidos. O índice de acurácia variou de $56,4 \%$ a $90,4 \%$ e de $55 \%$ a $96,2 \%$ entre meninas e meninos, respectivamente (Tabela 2).

Em caráter complementar os dados foram examinados segundo dependência administrativa da escola. Os resultados encontrados para a população geral foram mantidos (Tabela 3 ).

O tempo semanal mediano despendido com a prática de atividade física estimado pelos R24h foi superior para o grupo classificado como positivo para os indicadores ATIVO-300MIN e ATIVO-150MIN estimados pelo QUEST, quando comparado com aquele classificado como negativo para estes indicadores. A mediana do tempo semanal despendido na realização de atividade física dos adolescentes que praticam alguma atividade foi mais do que quatro vezes a observada para o grupo de indivíduos classificados como inativos. Os resultados foram mantidos com a estratificação segundo sexo e dependência administrativa da escola (Tabela 4).

A avaliação da concordância das estimativas do tempo total despendido com a prática de atividade física segundo o QUEST e os três R24h, conforme proposto por Bland \& Altman 26,27 , apontou que a média da diferença de tempo foi 34 minutos, com limite superior e inferior de concordância, respectivamente, de 394 e -325 minutos. Indicou, também, que quase todos os valores da diferença entre os métodos estavam localizados dentro do intervalo de concordância aceitável ( \pm 2 desvios-padrão).

\section{Discussão}

No presente estudo observou-se que os três indicadores referentes à prática semanal de atividades físicas utilizados pela PeNSE apresentam alta acurácia. Em geral, os indicadores referentes à prática de atividade física apresentaram melhor desempenho que os de comportamento sedentário, tanto em relação às estimativas de frequência quanto para os parâmetros de validade isolados (sensibilidade e especificidade) e combinado (índice de acurácia). Além disso, vale destacar que houve diferença da mediana do tempo semanal despendido com a prática de atividade física entre os adolescentes classificados como positivo e negativo pelos indicadores ATIVO-300MIN, ATIVO-150MIN e INATIVO, sugerindo bom poder discriminatório destes indicadores.

Para o conjunto de alunos estudados, com exceção dos indicadores INATIVO e tempo jogando videogame-2ha, que apresentaram maior especificidade, os valores de sensibilidade foram em geral, superiores aos de especificidade. Isso indica que eles foram mais eficientes na identificação dos adolescentes que realmente despendiam o tempo avaliado com a prática das atividades mensuradas, ou seja, que estavam expostos aos fatores de risco (atividades sedentárias) e de proteção (prática de atividades físicas) examinados na PeNSE. Por outro lado, eles foram menos eficientes em conferir classificação "negativa" àqueles que, de fato, não estavam expostos a esses fatores. Ainda assim, cabe destacar que, em geral, os valores do índice de acurácia foram elevados (superiores a 70\%), refletindo resultados razoáveis no que tange à validade dos indicadores utilizados pela PeNSE.

A comparação dos nossos resultados com os estudos de validação dos outros sistemas de vigilância dirigidos a adolescentes existentes no mundo deve ser realizada com cautela, em função de diferenças nas características da popula- 
Frequência (\%) de indicadores de atividade física segundo questionário (QUEST) da Pesquisa Nacional de Saúde do Escolar (PeNSE) e três recordatórios de 24 horas (R24h), sensibilidade, especificidade e índice de acurácia do QUEST em relação ao R24h. Escolares do 9o ano do Ensino Fundamental no Município do Rio de Janeiro, Brasil, 2011.

\begin{tabular}{|c|c|c|c|c|c|c|}
\hline Indicadores & QUEST & R24h & Valor de $p$ * & $\begin{array}{c}\text { Sensibilidade } \\
\text { QUEST (\%) }\end{array}$ & $\begin{array}{c}\text { Especificidade } \\
\text { QUEST (\%) }\end{array}$ & $\begin{array}{c}\text { Índice de acurácia } \\
\text { QUEST (\%) }\end{array}$ \\
\hline \multicolumn{7}{|l|}{ Prática de atividades físicas } \\
\hline ATIVO-300MIN ** & 52,0 & 45,0 & 0,104 & 77,9 & 69,1 & 73,1 \\
\hline 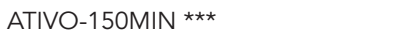 & 78,9 & 66,1 & 0,324 & 88,3 & 48,8 & 78,4 \\
\hline INATIVO \# & 1,8 & 7,0 & 0,022 & 8,3 & 98,7 & 92,4 \\
\hline \multicolumn{7}{|l|}{ Comportamento sedentário } \\
\hline Tempo assistindo TV-2ha \#\# & 83,8 & 84,4 & 1,000 & 90,4 & 51,9 & 84,4 \\
\hline Tempo assistindo TV-2hb \#\#\# & 83,8 & 60,1 & 0,000 & 92,3 & 29,0 & 67,1 \\
\hline Tempo jogando videogame-2ha \#\# & 19,7 & 8,1 & 0,001 & 50,0 & 83,0 & 80,3 \\
\hline Tempo jogando videogame-2hb \#\#\# & 19,7 & 1,2 & 0,000 & 100,0 & 81,3 & 81,5 \\
\hline Tempo usando computador-2ha \#\# & 69,5 & 58,0 & 0,006 & 86,1 & 53,4 & 72,4 \\
\hline Tempo usando computador-2hb \#\#\# & 69,5 & 27,6 & 0,000 & 95,8 & 40,5 & 55,7 \\
\hline
\end{tabular}

* Teste McNemar;

** $\geq 300$ minutos por semana;

*** $\geq 150$ minutos por semana;

\# Não praticou qualquer tipo de atividade física;

\#\# Pelo menos duas horas por dia, considerando-se que a condição foi atendida em pelo menos um dos dois R24h referentes a dias de semana;

\#\#\# Pelo menos duas horas por dia, considerando-se que a condição foi atendida nos dois R24h referentes a dias de semana.

ção estudada, no método de referência adotado e na análise estatística empregada. Além disso, os instrumentos adotados para aferir o nível de atividade física e comportamentos sedentários são distintos, com diferentes pontos de corte, o que limita ainda mais a comparabilidade dos estudos que avaliam o nível de atividade física de adolescentes 10,28,29.

Embora existam diferenças metodológicas, o presente estudo e a pesquisa que avaliou o desempenho do questionário de atividade física do Health Behaviour in School-aged Children (HBSC) para adolescentes australianos 17 apresentaram resultados satisfatórios. Assim como os indicadores ATIVO-300MIN e ATIVO-150MIN foram capazes de discriminar os adolescentes em relação ao tempo despendido com a realização de atividade física, os alunos australianos ativos, classificados segundo indicador utilizado pelo HBSC, apresentaram capacidade aeróbica significativamente maior do que os considerados insuficientemente ativos 17 . Já entre adolescentes noruegueses, o desempenho dos indicadores gerados pelo questionário do HBSC foi satisfatório para avaliar a capacidade cardiorrespiratória somente de meninas 20 .

Comparando-se os nossos achados com os do Youth Risk Behavior Surveillance System (YRBSS) na Califórnia, Estados Unidos 18, os valores de sensibilidade (80\%) e do índice de acurácia (73\%) do indicador ATIVO-300MIN aqui descritos foram superiores aos encontrados para o indicador daquele sistema $(71 \%$ e $63 \%$, respectivamente). Em relação às atividades de comportamento sedentário, embora o estudo de validação do questionário resumido sobre comportamentos sedentários do YRBSS realizado em Minnesota, Estados Unidos, tenha utilizado as variáveis de forma contínua, a comparação com o presente estudo é pertinente. O bom desempenho do indicador referente ao tempo despendido assistindo TV foi observado nos dois estudos. A similaridade entre os resultados não foi observada para o indicador relacionado ao tempo utilizando o computador, pois somente no presente trabalho foi observada diferença nas estimativas geradas pelos dois métodos 30 .

Em caráter complementar foi apresentada a comparação dos resultados do presente trabalho com os do estudo de validação dos indicadores de atividade física e de comportamento sedentário, utilizados pelo Sistema de Vigilância de Fatores de Risco e Proteção para Doenças Crônicas por Telefone (VIGITEL) para a população adulta de São Paulo, Brasil 22. Embora a validação de instrumentos e indicadores seja específica para cada contexto (por exemplo: gru- 
Tabela 2

Frequência (\%) de indicadores de atividade física segundo questionário (QUEST) da Pesquisa Nacional de Saúde do Escolar (PeNSE) e três recordatórios de 24 horas (R24h), sensibilidade, especificidade e índice de acurácia do QUEST em relação ao R24h segundo sexo. Escolares do 9o ano do Ensino Fundamental no Município do Rio de Janeiro, Brasil, 2011.

\begin{tabular}{|c|c|c|c|c|c|c|}
\hline Indicadores & QUEST & R24h & Valor de $p$ * & $\begin{array}{l}\text { Sensibilidade } \\
\text { QUEST (\%) }\end{array}$ & $\begin{array}{c}\text { Especificidade } \\
\text { QUEST (\%) }\end{array}$ & $\begin{array}{c}\text { Índice de acurácia } \\
\text { QUEST (\%) }\end{array}$ \\
\hline \multicolumn{7}{|c|}{ Prática de atividades físicas } \\
\hline \multicolumn{7}{|c|}{ ATIVO-300MIN ** } \\
\hline Meninas & 37,0 & 31,5 & 0,424 & 65,5 & 76,2 & 72,8 \\
\hline Meninos & 69,6 & 60,8 & 0,189 & 85,4 & 54,8 & 73,4 \\
\hline \multicolumn{7}{|c|}{ ATIVO-150MIN *** } \\
\hline Meninas & 69,6 & 60,9 & 0,152 & 85,7 & 55,6 & 73,9 \\
\hline Meninos & 89,9 & 91,1 & 1,000 & 90,3 & 14,3 & 83,5 \\
\hline \multicolumn{7}{|l|}{ INATIVO \# } \\
\hline Meninas & 3,3 & 9,8 & 0,109 & 11,1 & 97,6 & 89,1 \\
\hline Meninos & 0,0 & 3,8 & - & - & - & 96,2 \\
\hline \multicolumn{7}{|c|}{ Comportamento sedentário } \\
\hline \multicolumn{7}{|c|}{ Tempo assistindo TV-2ha \#\# } \\
\hline Meninas & 83,0 & 83,0 & 1,000 & 85,9 & 31,3 & 76,6 \\
\hline Meninos & 84,8 & 86,1 & 1,000 & 95,6 & 81,8 & 93,7 \\
\hline \multicolumn{7}{|c|}{ Tempo assistindo TV-2hb \#\#\# } \\
\hline Meninas & 83,0 & 57,4 & 0,000 & 88,9 & 25,0 & 61,7 \\
\hline Meninos & 84,8 & 63,3 & 0,000 & 96,0 & 34,5 & 62,7 \\
\hline \multicolumn{7}{|c|}{ Tempo jogando videogame-2ha \#\# } \\
\hline Meninas & 11,7 & 2,1 & 0,004 & 100,0 & 90,2 & 90,4 \\
\hline Meninos & 29,1 & 15,2 & 0,043 & 41,7 & 73,1 & 68,4 \\
\hline \multicolumn{7}{|c|}{ Tempo jogando videogame-2hb \#\#\# } \\
\hline Meninas & 11,7 & 0,0 & - & - & - & 88,3 \\
\hline Meninos & 29,1 & 2,5 & 0,000 & 100,0 & 72,7 & 73,4 \\
\hline \multicolumn{7}{|c|}{ Tempo usando computador-2ha \#\# } \\
\hline Meninas & 73,4 & 61,7 & 0,052 & 86,2 & 47,2 & 71,3 \\
\hline Meninos & 65,0 & 53,8 & 0,078 & 86,0 & 59,5 & 80,8 \\
\hline \multicolumn{7}{|c|}{ Tempo usando computador-2hb \#\#\# } \\
\hline Meninas & 73,4 & 31,9 & 0,000 & 96,7 & 37,5 & 56,4 \\
\hline Meninos & 65,0 & 22,5 & 0,000 & 94,4 & 43,5 & 55,0 \\
\hline
\end{tabular}

* Teste McNemar;

** $\geq 300$ minutos por semana;

$\star \star \star ~ \geq 150$ minutos por semana;

\# Não praticou qualquer tipo de atividade física;

\#\# Pelo menos duas horas por dia, considerando-se que a condição foi atendida em pelo menos um dos dois R24h referentes a dias de semana;

\#\#\# Pelo menos duas horas por dia, considerando-se que a condição foi atendida nos dois R24h referentes a dias de semana.

po populacional), essa comparação é oportuna na perspectiva de sistematizar a experiência de validação dos indicadores utilizados pelos sistemas brasileiros de vigilância de fatores de risco dirigidos a diferentes grupos populacionais. Os três indicadores aqui cotejados são INATIVO, ATIVO-150MIN e tempo assistindo TV-2ha, sendo que, no estudo referente ao VIGITEL, o ponto de corte adotado para o indicador referente ao tempo em frente à TV foi de três horas diárias em cinco ou mais dias da semana. Para esse último indicador, o desempenho foi similar nos dois estudos: ausência de diferença estatisticamente significativa na frequência estimada pelos métodos teste e de referência. Para os outros dois, entretanto, os resultados divergiram: no presente estudo, para o conjunto do grupo estudado, as frequências estimadas pelos métodos teste 
Frequência (\%) de indicadores de atividade física segundo questionário (QUEST) da Pesquisa Nacional de Saúde do Escolar (PeNSE) e três recordatórios de 24 horas (R24h), sensibilidade, especificidade e índice de acurácia do QUEST em relação ao R24h segundo dependência administrativa da escola. Escolares do 9o ano do Ensino Fundamental no Município do Rio de Janeiro, Brasil, 2011.

\begin{tabular}{|c|c|c|c|c|c|c|}
\hline Indicadores & QUEST & R24h & Valor de $p$ * & $\begin{array}{c}\text { Sensibilidade } \\
\text { QUEST (\%) }\end{array}$ & $\begin{array}{c}\text { Especificidade } \\
\text { QUEST (\%) }\end{array}$ & $\begin{array}{c}\text { Índice de acurácia } \\
\text { QUEST (\%) }\end{array}$ \\
\hline \multicolumn{7}{|c|}{ Prática de atividades físicas } \\
\hline \multicolumn{7}{|l|}{ ATIVO-300MIN ** } \\
\hline Escola pública & 44,7 & 35,1 & 0,052 & 76,3 & 94,8 & 76,3 \\
\hline Escola privada & 66,7 & 64,9 & 1,000 & 66,7 & 50,0 & 66,7 \\
\hline \multicolumn{7}{|l|}{ ATIVO-150MIN *** } \\
\hline Escola pública & 74,6 & 69,3 & 0,327 & 87,3 & 54,3 & 77,2 \\
\hline Escola privada & 87,7 & 86,0 & 1,000 & 89,8 & 25,0 & 80,7 \\
\hline \multicolumn{7}{|l|}{ INATIVO \# } \\
\hline Escola pública & 0,9 & 7,9 & 0,008 & 11,1 & 100,0 & 93,0 \\
\hline Escola privada & 3,5 & 5,3 & 1,000 & 0,0 & 96,3 & 91,2 \\
\hline \multicolumn{7}{|c|}{ Comportamento sedentário } \\
\hline \multicolumn{7}{|c|}{ Tempo assistindo TV-2ha \#\# } \\
\hline Escola pública & 87,2 & 85,5 & 0,815 & 92,0 & 41,2 & 84,6 \\
\hline Escola privada & 76,8 & 82,1 & 0,508 & 87,0 & 70,0 & 83,9 \\
\hline \multicolumn{7}{|c|}{ Tempo assistindo TV-2hb \#\#\# } \\
\hline Escola pública & 87,2 & 60,7 & 0,000 & 94,4 & 23,9 & 66,7 \\
\hline Escola privada & 76,8 & 58,9 & 0,031 & 87,9 & 39,1 & 67,9 \\
\hline \multicolumn{7}{|c|}{ Tempo jogando videogame-2ha \#\# } \\
\hline Escola pública & 17,9 & 8,5 & 0,028 & 50,0 & 85,0 & 82,1 \\
\hline Escola privada & 23,2 & 7,1 & 0,022 & 50,0 & 78,8 & 76,8 \\
\hline \multicolumn{7}{|c|}{ Tempo jogando videogame-2hb \#\#\# } \\
\hline Escola pública & 17,9 & 0,9 & 0,000 & 100,0 & 82,8 & 82,9 \\
\hline Escola privada & 23,2 & 1,8 & 0,000 & 100,0 & 78,2 & 78,6 \\
\hline \multicolumn{7}{|c|}{ Tempo usando computador-2ha \#\# } \\
\hline Escola pública & 64,1 & 60,7 & 0,597 & 80,3 & 60,9 & 72,6 \\
\hline Escola privada & 80,4 & 52,6 & 0,000 & 100,0 & 40,7 & 71,9 \\
\hline \multicolumn{7}{|c|}{ Tempo usando computador-2hb \#\#\# } \\
\hline Escola pública & 64,1 & 29,9 & 0,000 & 94,3 & 48,8 & 62,4 \\
\hline Escola privada & 80,4 & 22,8 & 0,000 & 100,0 & 25,0 & 42,1 \\
\hline
\end{tabular}

* Teste McNemar;

** $\geq 300$ minutos por semana;

$\star \star \star ~ \geq 150$ minutos por semana;

\# Não praticou qualquer tipo de atividade física;

\#\# Pelo menos duas horas por dia, considerando-se que a condição foi atendida em pelo menos um dos dois R24h referentes a dias de semana

\#\# Pelo menos duas horas por dia, considerando-se que a condição foi atendida nos dois R24h referentes a dias de semana.

e de referência foram distintas para o indicador INATIVO e semelhantes para o indicador ATIVO150MIN; no estudo de validação do questionário do VIGITEL observou-se o inverso.

Além disso, outra diferença foi observada: os valores de especificidade foram superiores aos de sensibilidade para os três indicadores avaliados pelo VIGITEL; no presente estudo, os valores de sensibilidade foram maiores que os de especi- ficidade para dois deles: ATIVO-150MIN e tempo assistindo TV-2ha. Cabe ressaltar, contudo, que em ambos os estudos foi observada diferença estatisticamente significativa da mediana do tempo total despendido com a prática de atividades físicas calculado pelo método de referência entre os indivíduos classificados pelo método teste como positivos e negativos para o indicador ATIVO150MIN 22. 
Mediana do tempo semanal despendido na realização de atividade física, em minutos, estimada pelos três recordatórios de 24 horas (R24h) para os adolescentes classificados, segundo o questionário (QUEST) da Pesquisa Nacional de Saúde do Escolar (PeNSE), como positivos ou negativos para cada indicador de prática de atividade física segundo sexo e dependência administrativa da escola. Escolares do 9o ano do Ensino Fundamental no Município do Rio de Janeiro, Brasil, 2011.

\begin{tabular}{|c|c|c|c|}
\hline \multirow[t]{2}{*}{ Indicadores } & \multicolumn{2}{|c|}{$\begin{array}{l}\text { Mediana do tempo despendido na prática de atividade } \\
\text { física estimado pelos R24h em uma semana (minutos) }\end{array}$} & \multirow[t]{2}{*}{ Valor de p * } \\
\hline & $\begin{array}{l}\text { Positivo para o indicador } \\
\text { segundo o QUEST }\end{array}$ & $\begin{array}{c}\text { Negativo para o indicador } \\
\text { segundo o QUEST }\end{array}$ & \\
\hline \multicolumn{4}{|l|}{ ATIVO-300MIN ** } \\
\hline Meninas & 319 & 106 & $<0,001$ \\
\hline Meninos & 438 & 293 & $<0,001$ \\
\hline Escola pública & 363 & 258 & $<0,001$ \\
\hline Escola privada & 411 & 238 & $<0,001$ \\
\hline Total & 392 & 150 & $<0,001$ \\
\hline \multicolumn{4}{|l|}{ ATIVO-150MIN *** } \\
\hline Meninas & 269 & 56 & $<0,001$ \\
\hline Meninos & 380 & 184 & $<0,001$ \\
\hline Escola pública & 275 & 50 & $<0,001$ \\
\hline Escola privada & 374 & 175 & $<0,001$ \\
\hline Total & 313 & 88 & $<0,001$ \\
\hline \multicolumn{4}{|l|}{ Inativo \# } \\
\hline Meninas & 63 & 163 & $<0,001$ \\
\hline Meninos & - & 365 & - \\
\hline Escola pública & 0,0 & 200 & $<0,001$ \\
\hline Escola privada & 224 & 363 & $<0,001$ \\
\hline Total & 63 & 275 & $<0,001$ \\
\hline
\end{tabular}

* Teste Wilcoxon para comparação de medianas dos grupos;

$\star \star \geq 300$ minutos por semana;

*** $\geq 150$ minutos por semana;

\# Não praticou qualquer tipo de atividade física.

Uma das possíveis limitações do presente estudo é o fato de o R24h ter sido usado como método de referência, uma vez que pode estar sujeito ao mesmo tipo de erro que o do método teste; por exemplo, imprecisão na estimativa do tempo alocado em cada atividade de interesse. No presente estudo, foram adotados três R24h como método de referência, em consonância com diversos estudos de validação de indicadores de prática de atividade física 22,31,32,33. Por motivos operacionais, não foi possível a adoção de métodos que não apresentam erros correlacionados como, por exemplo, acelerômetro, pedômetro, monitor da frequência cardíaca, água duplamente marcada, observação direta do comportamento e medida da aptidão cardiorrespiratória 29,34. Pelos mesmos motivos não foi possível a aplicação de sete R24h para caracterizar mais adequadamente a prática de atividade física $35 \mathrm{ou}$, ainda, a adoção conjugada do R24h com um método direto de aferição da atividade física 29,34 . Vale lembrar que problemas com a precisão do método de referência adotado não produzem superestimação da validade do método ou instrumento em questão, mas, sim, subestimação das medidas de validade avaliadas 22 .

No presente trabalho, os métodos de referência adotados para exposição aos comportamentos sedentários foram baseados em R24h referente(s) a dia(s) de semana típico(s) (segunda a sexta-feira). Em revisão sistemática que avaliou as formas de mensurar a exposição ao comportamento sedentário assistir televisão entre crianças e adolescentes, destacou-se que a maioria dos estudos avalia o tempo despendido assistindo televisão com perguntas autorrelatadas e não apresenta resultados de confiabilidade e validade. Os poucos instrumentos submetidos a estudos de 
validação foram comparados com métodos de referência baseados em autorrelato (registro ou recordatório) ou em alguma medida direta de atividade. Nenhum estudo utilizou a observação direta (pelo pesquisador ou gravada em vídeo), considerada padrão ouro, para avaliar a atividade em questão 10. Em outra revisão sistemática sobre a validade de instrumentos que aferem comportamentos sedentários entre adultos, destacou-se que o instrumento com maior validade foi o que avaliou questões sobre um dia típico 36 .

Uma vantagem do método de referência adotado é o fato de terem sido realizadas entrevistas aos sábados e domingos para aplicar os R24h referentes aos dias de sexta-feira e sábado, respectivamente, o que permitiu representar igualmente os dias da semana. Isso foi possível porque as entrevistas foram realizadas por telefone. Esse é um diferencial do estudo, uma vez que, comumente, em pesquisas dirigidas a escolares, a coleta de dados é realizada na própria escola, entre segunda e sexta-feira 17,37.

A sequência de aplicação dos instrumentos pode influenciar os resultados de um estudo de validação ${ }^{16}$. O método a ser testado deve ser administrado primeiro para evitar que a realização do método de referência influencie o resultado do instrumento a ser avaliado. Para garantir a adequada sequência de aplicação, o estudo comparou duas semanas distintas. A variação real do nível de atividade física e da exposição aos comportamentos sedentários pode ser responsável por parte das diferenças observadas entre os dois métodos. Para reduzir essa diferença, os três R24h foram aplicados no menor intervalo de tempo possível.

Comparando-se o desempenho do QUEST com as duas formas de se estimar o tempo despendido em comportamentos sedentários, observou-se que os resultados do QUEST ficaram mais próximos aos obtidos utilizando-se a primeira forma (a condição ter sido atendida em pelo menos um dos dois R24h) quando comparados àqueles obtidos utilizando-se a segunda forma (a condição ter sido atendida nos dois R24h). Cabe aqui problematizar a limitação da utilização de informações pontuais (um ou dois dias) para representar um dia de semana comum. Quando são avaliados dois dias e eles são diferentes, não se sabe qual deles é o típico. Uma opção para contornar isso em estudos futuros, quando não for possível avaliar os sete dias da semana, seria realizar três recordatórios referentes a dias de semana e considerar como típica a situação que acontecesse em pelo menos dois destes dias.

Os indicadores referentes à inatividade física e ao tempo alocado no uso de videogame apre- sentaram valores extremos de sensibilidade e especificidade em parte das análises realizadas. Isso pode ser devido ao fato de eles terem apresentado frequência porcentual de ocorrência inferior à utilizada como parâmetro para o cálculo da amostra. Por esse motivo, pode-se supor que o tamanho da amostra não tenha sido suficiente para estimar com mais precisão esses parâmetros de validade dos indicadores.

Ainda em relação aos comportamentos sedentários, a inclusão de questões referentes ao fim de semana no questionário da PeNSE, possivelmente, faria com que o desempenho dos indicadores melhorasse. As outras questões do bloco de atividade física do questionário são referentes aos sete dias anteriores à pesquisa. Como, no presente estudo, os resultados dos indicadores de comportamento sedentário, que se referem à rotina durante a semana, foram superestimados pelo QUEST, uma possibilidade seria a de que os adolescentes tenham levado em consideração o tempo despendido com essas atividades também nos fins de semana, assim como fizeram para todas as outras perguntas sobre atividade física.

Para permitir a extrapolação dos resultados de um estudo de validação, os indivíduos participantes de uma pesquisa devem apresentar variabilidade das medidas de interesse semelhantes à da população na qual o instrumento será aplicado. Um instrumento pode ser considerado válido para um grupo populacional com características como nível educacional ou socioeconômico semelhante àquelas do grupo avaliado no estudo de validação. Caso contrário, é necessário realizar novos estudos para avaliar a validade do instrumento nas diferentes circunstâncias 16. Os resultados do presente estudo não são, necessariamente, extrapoláveis para o país como um todo. Nesse sentido, um aspecto a ser considerado é o fato de que a proporção de alunos de escolas particulares observada na PeNSE de 2009 para o Rio de Janeiro e para o conjunto de capitais brasileiras e o Distrito Federal $(23,4 \%$ e $20,8 \%$, respectivamente) foi inferior à observada no presente estudo (32,8\%). Essa diferença pode ser justificada pelo fato de a proporção de alunos em escolas públicas e particulares ser diferente da observada para o município no Censo Escolar de 2010, que foi utilizado como base para o sorteio da amostra. Embora tenha havido maior número de perdas entre os alunos de escolas particulares, esse estrato ficou sobrerrepresentado no estudo. Entretanto, o fato de os resultados terem se mantido quando os dados foram analisados segundo dependência administrativa da escola fala a favor da validade externa do estudo para realidades semelhantes às do Rio de Janeiro. 
O presente trabalho, no qual foi avaliado uma quantidade expressiva de indicadores de atividade física e comportamento sedentário, traz contribuição pioneira para o sistema brasileiro de vigilância de fatores de risco para a saúde de adolescentes. Seus resultados sugerem que os indicadores de atividade física utilizados pela PeNSE apresentaram desempenho satisfatório quando avaliados no Município do Rio de Janeiro. A realização de estudos similares em outras localidades do país permitirá maior conhecimento sobre a validade desses indicadores utilizados pelo sistema de vigilância de fatores de risco para a saúde de adolescentes brasileiros.

\section{Resumen}

Se analizó la validez relativa de los indicadores de actividad física del cuestionario utilizado en la Encuesta Nacional de Salud Escolar (PeNSE por sus siglas en portugués) entre adolescentes en la ciudad de Río de Janeiro, Brasil. Se estudiaron 174 alumnos. Fueron evaluados los siguientes indicadores, referidos al tiempo semanal acumulado con actividad física: ACTIVO300MIN (acúmulo de 300 minutos o más); ACTIVO150MIN (acúmulo de 150 minutos o más), INACTIVO (no practica actividad física). También se han estudiado indicadores de conducta sedentaria como: tiempo diario viendo televisión, jugando videojuegos y usando la computadora. Se compararon los resultados del cuestionario y los tres recordatorios de 24 horas. Los resultados de ACTIVO-300MIN, ACTIVO-150MIN e INACTIVO, generados por PeNSE, mostraron una alta precisión. Estos indicadores se comportaron mejor que los de comportamiento sedentario, tanto con respecto a la estimativa de frecuencia, como a la sensibilidad, la especificidad e índice de precisión. Los indicadores de actividad física de PeNSE presentaron validez relativa satisfactoria.

Actividad Motora; Estudios de Validación; Estilo de Vida Sedentario; Adolescente; Vigilancia

\section{Colaboradores}

L. F. Tavares e I. R. R. Castro contribuíram no delineamento do estudo, na supervisão do trabalho de campo, na análise do banco de dados e na redação do manuscrito. L. O. Cardoso e R. B. Levy participaram do delineamento do estudo e na revisão do manuscrito. R. M. Claro colaborou na análise do banco de dados e na revisão do manuscrito. A. F. Oliveira contribuiu na supervisão do trabalho de campo e na revisão do manuscrito.

\section{Agradecimentos}

Ao Instituto de Nutrição Annes Dias pelo apoio logístico para a realização do trabalho de campo. À Faperj (processo E-26/110.635/2011) e CNPq (processo 475782/2011-9) pelo financiamento e ao Instituto Brasileiro de Geografia e Estatística (IBGE) pelo empréstimo dos equipamentos PDA. 


\section{Referências}

1. World Health Organization. Global recommendations on physical activity for health. Geneva: World Health Organization; 2010.

2. World Health Organization. Global recommendations on physical activity for health: 5-17 years old. Geneva: World Health Organization; 2011.

3. Hallal PC, Victora CG, Azevedo MR, Wells JCK. Adolescent physical activity and health: a systematic review. Sports Med 2006; 36:1019-30.

4. Hallal PC, Simões E, Reichert FF, Azevedo MR, Ramos LR, Pratt M, et al. Validity and reliability of the Telephone-Administered International Physical Activity Questionnaire in Brazil. J Phys Act Health 2010; 7:402-9.

5. Tenório MCM, Barros MVG, Tassitano RM, Bezerra J, Tenório JM, Hallal PC. Atividade física e comportamento sedentário em adolescentes estudantes do ensino médio. Rev Bras Epidemiol 2010; 13:105-17.

6. Guthold R, Cowan MJ, Autenrieth CS, Kann L, Riley LM. Physical activity and sedentary behavior among schoolchildren: a 34-country comparison. J Pediatr 2010; 157:43-9.

7. Eaton DK1, Kann L, Kinchen S, Shanklin S, Flint $\mathrm{KH}$, Hawkins J, et al. Youth risk behavior surveillance - United States, 2011. MMWR Surveill Summ 2012; 61:1-162.

8. Currie C, Zanotti C, Morgan A, Currie D, Looze $\mathrm{M}$, Roberts C, et al. Social determinants of health and well-being among young people. Health Behaviour in School-aged Children (HBSC) study: international report from the 2009/2010 survey. Geneva: World Health Organization; 2012.

9. Hancox RJ, Milne BJ, Poulton R. Association between child and adolescent television viewing and adult health: a longitudinal birth cohort study. Lancet 2004; 364:257-62.

10. Bryant MJ, Lucove JC, Evenson KR, Marshall S. Measurement of television viewing in children and adolescents: a systematic review. Obes Rev 2007; 8:197-209

11. Cardoso LO. Fatores associados ao excesso de peso e perfis de consumo e comportamento alimentar de adolescentes [Tese de Doutorado]. Rio de Janeiro: Escola Nacional de Saúde Pública Sérgio Arouca, Fundação Oswaldo Cruz; 2010.

12. Brener ND, Kann L, Kinchen SA, Grunbaum JA, Whalen L, Eaton D, et al. Methodology of the youth risk behavior surveillance system. MMWR Recomm Rep 2004; 53:1-13.

13. Currie C, Roberts C, Morgan A, Smith R, Settertobulte W, Samdal O, et al. Young people's health in context: Health Behaviour in School-aged Children (HBSC) study: international report from the 2001/2002 survey. Geneva: World Health Organization; 2004.

14. Centers for Disease Control and Prevention. Global School-based Student Health Survey (GSHS). http://www.cdc.gov/gshs/background/index. htm\#2 (acessado em 28/Ago/2012).
15. Instituto Brasileiro de Geografia e Estatística. Pesquisa Nacional de Saúde Escolar. Rio de Janeiro: Instituto Brasileiro de Geografia e Estatística; 2009.

16. Streiner DL, Norman GR. Health measurement scales: a practical guide to their development and use. 4th Ed. New York: Oxford University Press; 2008.

17. Booth ML, Okely AD, Chey T, Bauman A. The reliability and validity of the physical activity questions in the WHO health behaviour in schoolchildren (HBSC) survey: a population study. Br J Sports Med 2001; 35:263-7.

18. Prochaska JJ, Sallis JF, Long B. A physical activity screening measure for use with adolescents in primary care. Arch Pediatr Adolesc Med 2001; 155:554-9.

19. Troped PJ, Fragala MS, Finkelstein DM, Kim J, Peterson KE. Reliability and validity of YRBS physical activity items among middle school students. Med Sci Sports Exerc 2007; 39:416-25.

20. Rangul V, Holmen TL, Kurtze N, Cuypers K, Midthjell K. Reliability and validity of two frequently used self-administered physical activity questionnaires in adolescents. BMC Med Res Methodol 2008; 8:47.

21. Reichenheim ME. Sample size for the kappa-statistic of interrater agreement. Stata Technical Bulletin 2000; 58:41-5.

22. Monteiro CA, Florindo AA, Claro RM, Moura EC. Validade de indicadores de atividade física e sedentarismo obtidos por inquérito telefônico. Rev Saúde Pública 2008; 42:575-81.

23. Castro IRR, Cardoso LO, Egstrom EM, Levy RB, Monteiro CA. Vigilância de fatores de risco para doenças não transmissíveis entre adolescentes: a experiência da cidade do Rio de Janeiro, Brasil. Cad Saúde Pública 2008; 24:2279-88.

24. Szklo M, Javier Nieto F. Epidemiology: beyond the basics. 2nd Ed. Frederick: Aspen Publishers; 2007.

25. Siqueira AL, Tibúrcio JD. Estatística na área da saúde: conceitos, metodologia, aplicações e prática computacional. Belo Horizonte: Coopmed; 2011.

26. Bland JM, Altman DG. Statistical methods for assessing agreement between two methods of clinical measurement. Lancet 1986; 1:307-10.

27. Bland JM, Altman DG. Comparing methods of measurement: why plotting difference against standard method is misleading. Lancet 1995; 346:1085-7.

28. Sallis JF, Saelens BE. Assessment of physical activity by self-report: status, limitations, and future directions. Res Q Exerc Sport 2000; 71:1-14.

29. Farias Júnior JC, Lopes AS, Florindo AA, Hallal PC. Validade e reprodutibilidade dos instrumentos de medida da atividade física tipo self-report em adolescentes: uma revisão sistemática. Cad Saúde Pública 2010; 26:1669-91. 
30. Schmitz KH, Harnack L, Fulton JE, Jacobs Jr. DR, Gao S, Lytle LA, et al. Reliability and validity of a brief questionnaire to assess television viewing and computer use by middle school children. J Sch Health 2004; 74:370-7.

31. Farias Júnior JC, Lopes AS, Mota J, Santos MP, Ribeiro JC, Hallal PC. Validade e reprodutibilidade de um questionário para medida de atividade física em adolescentes: uma adaptação do Self-Administered Physical Activity Checklist. Rev Bras Epidemiol 2012; 15:198-210.

32. Hernández B, Gortmaker SL, Laird NM, Colditz GA, Parra-Cabrera S, Peterson KE. Validez y reproducibilidad de un questionario de actividad e inactividad física para escolares de la ciudad de México. Salud Pública Méx 2000; 42:315-23.

33. Sallis JF, Strikmiller PK, Harsha D, Feldman HA. Validation of interviewer-and-self-administered physical activity checklists for fifth grade students. Med Sci Sports Exerc 1996; 28:840-51.
34. Adamo KB, Prince SA, Tricco AC, Connor-Gorber S, Tremblay M. A comparison of indirect versus direct measures for assessing physical activity in the pediatric population: a systematic review. Int J Pediatr Obes 2009; 4:2-27.

35. Sallis JE. Seven-day physical activity recall (1985). Med Sci Sports Exerc 1997; 29(6 Suppl):S89-103.

36. Clark BK, Sugiyama T, Salmon J, Dunstan DW, Owen N. Validity and reliability of measures of television viewing time and other non-occupational sedentary behaviour of adults: a review. Obes Rev 2009; 10:7-16.

37. Guedes DP, Lopes CC, Guedes JERP. Reprodutibilidade e validade do questionário internacional de atividade física em adolescentes. Rev Bras Med Esporte 2005; 11:151-8.

Recebido em 20/Ago/2013

Versão final reapresentada em 07/Jan/2014

Aprovado em 17/Fev/2014 